\title{
Effects of stride length and frequency training on acceleration kinematic, and jumping performances
}

\author{
Mehmet $\mathrm{KALE}^{1} \bullet$ Caner ACIKADA ${ }^{2}$
}

\begin{abstract}
$\mathrm{T}$ he purpose of this study was to investigate the effects of additional short and long running stride workouts on acceleration kinematics and jumping performances with respect to the competition phase. Thirteen voluntary male sprinters participated in this study (100 m PB: 10.45-11.30s). The sprinters were separated into three groups [control group (CG), short stride group (SSG), long stride group (LSG)] in a randomised order. Sprinters continued their competition phase training over a two-week period; however, the SSG and LSG run an additional $6 \times 40 \mathrm{~m}$ three times per week. The pre- and post-training results regarding, acceleration kinematics and jumping performances were compared using the Kruskal Wallis test for several independent samples of intergroup and the Wilcoxon Two Related Samples Test for intragroup differences. If there were intergroup differences, the Mann Whitney $U$ test was used for two related comparisons of the groups. The probability level for statistical significance was set to $\mathrm{p} \leq 0.05$. Only the LSG showed significant pre- and post-training differences with respect to certain acceleration kinematics (sprint time, sprint velocity, stride length) $(Z=-2.023$, $2.032,2.060$, respectively; $\mathrm{p}<0.05)$. In conclusion, the results of this study show that there is no significant effect of a two-week training regimen involving additional long and short stride running workouts on Turkish sprinters in the competition phase; however, long stride running workouts may be useful for sprinters who have short stride sprint characteristics.
\end{abstract}

Keywords: sprinter, competition phase, sprint, short stride, long stride

${ }^{1}$ Functional Movement Laboratory, Department of Coaching Education, Faculty of Sport Sciences, Anadolu University, Eskişehir, Turkey.

${ }^{2}$ School of Public Health, Indiana University, Bloomington, USA. 


\section{Introduction}

Complex training is necessary for rapid, explosive and powerful muscle contraction in sprint running. Adaptations to such complex training are manifested as the changes resulting from regular workouts. This type of training is programmed to optimise the benefits of chronic sprint workouts, but an awareness of the effects of the training regimen is required for these adaptations to be realised. Physiological and biomotor characteristics are specified, and training programs are planned around these characteristics utilising sportive performance tests under track and laboratory conditions.

The main goal in sprint training is to maximise speed and focus on power and explosiveness (Bushnell \& Hunter, 2007). High explosive muscle power, coordination, and ability are required for sprinters to execute fast acceleration before reaching maximal speed (Hawley, 2000). Stride length should be increased in the acceleration phase of sprinting. Therefore, sprinters have the ability to accelerate rapidly (Abe et al., 2001); however, they typically require sprint strength improvement, particularly in the start-acceleration phase. Effective acceleration is possible with high force-production skill throughout the contact time (CT) as progressive increases in stride length and frequency are made (Mann et al., 1984).

The acceleration phase in sprint running is biomechanically characterised by a forward leaning position with a $45^{\circ}$ angle between the leg and ground (Mann et al., 1984). A dominant horizontal force in effective acceleration is required to drive the lean forward. This force is mainly generated by muscular contractions rather than elastic power responses. Great body strength and power are needed to accelerate the body quickly and effectively. It has been reported that a $46 \%$ greater horizontal force is produced over a longer CT in the acceleration phase of sprinting than in the maximum speed phase (Mero, 1988). Moreover, EMG activity in the acceleration phase of the CT has been observed to be higher than that in the maximum speed phase. Thus, neuromuscular recruitment in which neural activity is reached at the maximum level during the acceleration phase is an important factor in the acceleration phase (Mero \& Peltola, 1981). It should be noted that stride frequency (SF) and stride length (SL) are improved at much different rates while attempting to reach maximum speed. Gradually decreasing long SLs helps increase CT and the horizontal force generated in this phase.

It is possible to determine the kinematics and physiological changes caused by training to identify the physical status of athletes after training and revise the performance status of athletes with a potential for over-training and injury. Evaluation of speed parameters, and jumps reveals important information about 
the training status of athletes. Which tests should be used and how often these tests should be performed to evaluate an athlete's current performance may vary according to the event or training program in which athletes participate. The parameters of sprint running performance and jumping performance and the effects of training with different running stride characteristics over the sprint running time are not widely addressed in detailed studies on the performance criteria of sprinters. Thus, the purpose of this study was to investigate the effects of a two-week training regimen involving $40 \mathrm{~m}$ of additional short and long running stride workouts on the acceleration kinematics and jumping performance of $100-\mathrm{m}$ sprinters.

\section{Material and methods}

\section{Participants}

Thirteen male Turkish Track and Field Cup 1 and 2 sprinters who had not suffered lower-extremity injuries over the past year participated in the study and performed regular strength/power training (more than three times per week for $\geq 2 \mathrm{yr}$ ). The best pre-request $100-\mathrm{m}$ performances of the subjects ranged between $10.45 \mathrm{~s}$ and $11.10 \mathrm{~s}$. Subjects were separated into three groups: CG [age (year): 22.0 $\pm 2.6,100 \mathrm{~m} \mathrm{~PB}(\mathrm{~s}): 10.93 \pm 0.23$, body height $(\mathrm{cm}): 177.4 \pm 3.3$, body weight (kg): $75.7 \pm 2.9$ ], SSG [age (year): $24.5 \pm 1.7,100 \mathrm{~m} \mathrm{~PB}(\mathrm{~s}): 10.75 \pm 0.14$, body height (cm): $175.4 \pm 3.2$, body weight $(\mathrm{kg}): 74.7 \pm 2.7$, and LSG [age (year): $20.4 \pm 2.1,100$ $\mathrm{m} \mathrm{PB}(\mathrm{s}): 11.06 \pm 0.17$, body height $(\mathrm{cm}): 176.3 \pm 2.3$, body weight $(\mathrm{kg}): 73.2 \pm 7.1]$ in a random order. Subjects were free to discontinue their participation in the study at any time, and they signed their statements of informed consent after the procedures and probable risks of the experiment were explained to them. The study was approved by the Osmangazi University Ethical Advisory Committee prior to the commencement of testing.

\section{Procedures}

Each sprinter was pre- and post-tested after laboratory and track visits lasting 45-60 min for each test protocol at a specific time on three occasions to complete familiarisation trials and the main trials. Subjects took part in tests including $100 \mathrm{~m}$ of sprint running to determine the kinematic parameters of sprint acceleration, vertical jumps [squat jump (SJs), countermovement jumps (CMJs), depth jumps (DJ)s], horizontal jumps [(standing long jumps (SLJs), standing triple jumps (STJs), standing quintuple jump (SQJs), standing ten jump (STENJ)s] over a two-day period (100m sprint tests in the first day, jump tests in the second day) in a random order. After the tests, sprinters were separated into three groups (CG, SSG, and LSG) in a random order. Each group continued their competition training. Participants in the SSG and LSG groups ran an additional $6 \times 40 \mathrm{~m}$ three times per week for two weeks. Each group was retested after the 
two-week training period. Pre- and post-training sprint running kinematics and differences in performance between the SSG and LSG due to additional short or long stride running workouts are presented in Table 1.

\begin{tabular}{lllll}
\hline Sprint parameters & Group & Pre & Post & Change Of rate $(\%)$ \\
\hline \multirow{2}{*}{$40 \mathrm{~m}_{\mathrm{t}}(\mathrm{s})$} & SSG & $5.15 \pm 0.11$ & $5.74 \pm 0.16$ & 11.6 \\
& LSG & $5.36 \pm 0.13$ & $6.55 \pm 0.24$ & 22.0 \\
$40 \mathrm{~m}_{\mathrm{v}}\left(\mathrm{m} \cdot \mathrm{s}^{-1}\right)$ & $\mathrm{SSG}$ & $7.77 \pm 0.16$ & $6.99 \pm 0.19$ & -10.0 \\
& LSG & $7.51 \pm 0.15$ & $6.15 \pm 0.22$ & -18.1 \\
$\mathrm{SL}(\mathrm{m})$ & $\mathrm{SSG}$ & $1.79 \pm 0.06$ & $1.45 \pm 0.05$ & -19.4 \\
& LSG & $1.76 \pm 0.11$ & $2.39 \pm 0.05$ & 36.0 \\
$\mathrm{SF}(\mathrm{Hz})$ & SSG & $4.63 \pm 0.33$ & $5.26 \pm 0.05$ & 13.5 \\
& LSG & $4.60 \pm 0.21$ & $2.82 \pm 0.17$ & -38.7 \\
TSL $(\mathrm{m})$ & SSG & $38.98 \pm 0.95$ & $39.55 \pm 0.22$ & 1.4 \\
& LSG & $39.59 \pm 0.47$ & $39.47 \pm 0.18$ & -0.3 \\
TSN (n) & SSG & $22 \pm 2$ & $28 \pm 1$ & 27.7 \\
& LSG & $23 \pm 2$ & $17 \pm 1$ & -26.5 \\
\hline
\end{tabular}

Data are means $\pm \mathrm{SD}$.

Table 1 - Pre- and post-training $40 \mathrm{~m}$ sprint running kinematics and change of rate $(\%)$ of SSG $(n=4)$ and $L S G(n=5)$

\section{Sprint Test}

The sprint running performance of sprinters was tested by a customised Precision Timing System (PTS) (Tümer Electronic Ltd, Turkey) starting with a signal for reaction. Interval timings of $10 \mathrm{~m}$ over a sprinting distance of 100 $\mathrm{m}$ were measured with a PTS consisting of two channels of data acquisition, an electronic receiver, and 10 infrared photocells. The height of the photocells was adjusted to the trochanter height of each subject to standardise the point at which infrared light crossed the subject's body. An ESC 2XXX Series Data Acquisition Software (Tümer Electronic Ltd, Turkey) was used to record the time data sampled at $\pm 0.01 \mathrm{~ms}$ to a portable computer via a serial slot (RS232) during the tests. The sprinters performed two $100-\mathrm{m}$ sprints on a synthetic field from starting blocks with 8-12 min of full recovery following a warm-up phase involving self-paced running, calisthenics and stretching exercises. The better trial was retained for statistical analysis. During the sprint tests and additional $6 \times 40 \mathrm{~m}$ of training, athletes wore tight-fitting clothes and spiked shoes. Each $\mathrm{V}_{10 \mathrm{~m}}$ in $\mathrm{m} \cdot \mathrm{s}^{-1}$ was calculated by formula 1 , where $\mathbf{d}_{\mathbf{x}}$ is the constant $10-\mathrm{m}$ interval distance and $\mathbf{t}$ is the $10-\mathrm{m}$ interval time in seconds. The acceleration phase parameters measured in the first $40 \mathrm{~m}$ before the highest $V_{10}$ of the best $100-\mathrm{m}$ sprint were retained for statistical analysis.

$$
\mathrm{V}_{\mathrm{x}}=\mathrm{d}_{\mathrm{x}} \cdot \mathrm{t}^{-1}
$$

[Formula-1] 


\section{Jump Test}

Time data (flight time for SJs and CMJs, flight time and CT for DJs) for SJs, CMJs, and DJs sampled at $1000 \mathrm{~Hz}$ were recorded using the software employed for the sprint test and saved on a portable computer. CT was recorded as the difference in time between the moment the timer of the PTS was triggered by the feet of each subject as he touched the switching mat and the moment of release from the mat. FT was recorded as the difference in time between the moment the PTS timer was triggered by the feet of the subject at the moment of release from the mat and the moment the subject touched down. Formula 2 (Bosco et al., 1983) was used to obtain all jump heights.

$$
\mathrm{h}=\mathrm{g} \cdot \mathrm{t}_{\mathrm{f}}^{2} \cdot \mathrm{8}^{-1} \cdot 100
$$
time (s).

$\mathrm{h}=$ jump height $(\mathrm{cm}) ; \mathrm{g}=$ gravitational acceleration $\left(9.80665{\left.\mathrm{~m} \cdot \mathrm{s}^{-2}\right)}^{-2} \mathrm{t}_{\mathrm{f}}=\right.$ flight

When performing SJs, the sprinters flexed their knees until they felt a comfortable starting position, a semi-squatting position, which normally occurs at a knee angle of approximately $85^{\circ}$ (Bosco \& Komi, 1979), and maintained their posture for at least two to three seconds; this prevented the pre-stretching of muscles from any preliminary downward movement before jumping. CMJs were performed in an upright standing position with hands kept on the hips following a preliminary downward movement by flexing the knee approximately to the same knee angle as the starting position in a SJ. The elastic power (CMJ$\mathrm{SJ}_{\text {dif }}$ ) was calculated as the difference between the power measured for the SJ and that measured for the CMJ. For DJs, sprinters were instructed to step from the DJ stair and perform a maximum effort vertical jump immediately after landing on the switching mat. The sprinters were kindly asked to land on the mat after the vertical jump. The position of the sprinters' centre of mass was nearly the same during take-off and landing, and the sprinters were asked to keep their hands on their hips throughout the movement. Subjects performed drop jumps from the DJ stair in a random order and were instructed to perform one trial at each drop height, with a rest period of $5 \mathrm{~min}$. CT and FT for each DJ were recorded to calculate the maximum jump height and jump power. The drop height resulted in a maximum jump height, which was used as the threshold drop jump height (TDJH) for statistical analysis. If the value of the maximum jump height was the same as another drop jump height, the jump having the highest power (calculated with Formula-3) (Bosco et al., 1983) was used.

$$
\mathrm{P}=\left(\mathrm{g}^{2} \cdot \mathrm{t}_{\mathrm{f}} \cdot \mathrm{t}_{\mathrm{t}}\right) \cdot\left(4 \cdot \mathrm{t}_{\mathrm{c}}\right)^{-1}
$$

$\mathrm{P}=$ mechanical power of one jump $\left(\mathrm{W}^{\mathrm{kg}} \mathrm{kg}^{-1}\right) ; \mathrm{t}_{\mathrm{c}}=$ contact time of one jump $(\mathrm{s}) ; \mathrm{t}_{\mathrm{t}}=$ total time of one jump (s). 


\section{Horizontal Jumps}

Sprinters performed two trials for each horizontal jump (SLJ, STJ, SQJ and STENJ) with a 5-min rest period in a random order. The longer horizontal distance of two trials for each type of jump was recorded for statistical analysis. A SLJ test was performed with the subjects' feet together and on the side of the long jump area of the test field and completed with both feet landing in the long jump area. SLJ distance, the distance between the feet at the starting line and the back trace of the sprinters in the long jump area, was recorded in $\mathrm{cm}$. A STJ test, consisting of three dynamic horizontal bounds, was used to assess rebound stretch shortening cycle muscular contractions. Each subject started from $6 \mathrm{~m}$ behind the long jump area with his feet together and performed two consecutive bounds using alternate feet. The third contact was made with the feet together, landing in the long jump area. The STJ distance was recorded as the distance between the landing points at the start line and the back trace of the sprinters in the long jump area in $\mathrm{cm}$. The SQJ tested started from $12 \mathrm{~m}$ behind the long jump area, and contact with the ground after the fifth bound was made with the feet together, landing in the long jump area after four consecutive bounds using alternate feet as in the SQJ procedure. The STENJ test started $25.5 \mathrm{~m}$ behind the long jump area, with 10 consecutive bounds performed and measured as in the STJ and SQJ tests.

\section{Two-Dimensional Motion Analysis of Sprint Running}

For digitisation, two markers were fixed on top of the fifth metatarsal of the right spiked shoe and first metatarsal of the left spiked shoe of each participant. The SIMI 7.3 motion analysis program (Germany) was utilised to analyse kinematics variables over $40 \mathrm{~m}$ of sprinting [ $\left(40-\mathrm{m}\right.$ time $\left(\mathrm{t}_{40 \mathrm{~m}}\right), 40-\mathrm{m}$ velocity $\left(\mathrm{V}_{40 \mathrm{~m}}\right)$, stride length (SL), stride frequency (SF), total stride length (TSL), total stride number (TSN)] in a 100-m sprint and also an additional 6 x $40 \mathrm{~m}$ of training with short strides and long strides. Two-dimensional foot motion over those $40 \mathrm{~m}$ was recorded at $80 \mathrm{~Hz}$ by four digitised video cameras (A602f, Basler, Germany) placed near the running line, as shown in Figure 1. The motion areas for each camera were calibrated using four points $(1.50 \mathrm{~m} \times 2.53 \mathrm{~m})$ within the SIMI calibration framework. Pointed markers for each recording frame for all $40 \mathrm{~m}$ were digitised, and two-dimensional calculations were performed. All coordinates were filtered by a second low-pass Butterworth digital filter (cut-off frequency of 5) to eliminate pointing errors in the raw data. SL and SF were calculated directly by filtering the time-position coordinates. To calculate $V_{40 \mathrm{~m}}$, the $40-\mathrm{m}$ distance was divided by $\mathrm{t}_{40 \mathrm{~m}}$. 


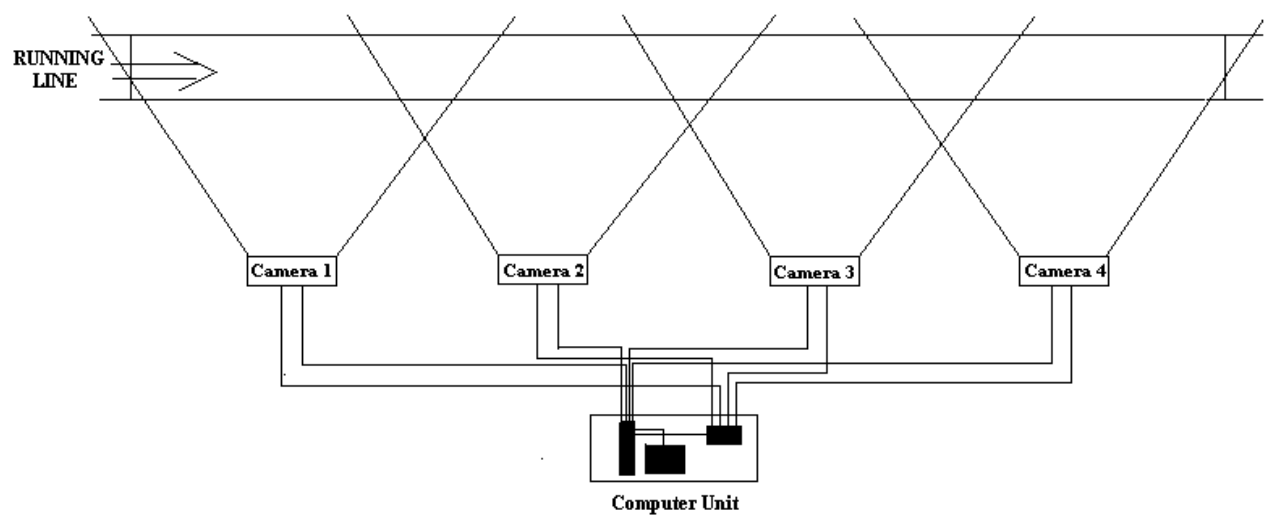

Figure 1 - Test design of two-dimensioned motion analysis of sprint running

\section{Measurement Track and Laboratory Conditions}

The wind conditions in each $100 \mathrm{~m}$-sprint test were measured with a wind gauge (0.01 m. $\mathrm{s}^{-1} \pm$; UCS Spirit Wind Gauge, USA) according to the wind criteria set by the IAAF for the 100-m sprint competition. The test was repeated when the wind exceeded the upper limit $\left(\geq 2.00 \mathrm{~m} . \mathrm{s}^{-1}\right)$. The track and laboratory temperature $\left(0.1 \pm^{\circ} \mathrm{C}\right)$ and humidity $(0.1 \% \pm)$ were measured with an anemometer (Traceable, Control Company, USA).

\section{Statistical Analysis}

SPSS 19 was used for statistical data analysis. Pre- and post-training differences of kinematic variables were analysed using the Kruskal Wallis test for several independent samples of intergroup and Wilcoxon Two Related Samples Test for intragroup differences. If there were intergroup differences, the Mann Whitney U test was used for two related comparisons of the groups. The probability level for statistical significance was set to $\mathrm{p} \leq 0.05$.

\section{Results}

Pre- and post-training means and standard deviations, changes in rate and intergroup and intragroup differences in the competition phase for acceleration kinematics, and jump parameters were presented Table 2, 3, and 4 . 
Effects of stride length and frequency training

\begin{tabular}{|c|c|c|c|c|c|}
\hline $\begin{array}{c}\text { Sprint } \\
\text { parameters }\end{array}$ & Groups & Pre & Post & $\begin{array}{c}\text { Change of } \\
\text { rate }(\%)\end{array}$ & Z \\
\hline \multirow{4}{*}{$\mathrm{t}_{40 \mathrm{~m}}(\mathrm{~s})$} & CG $(n=4)$ & $5.27 \pm 0.23$ & $5.30 \pm 0.19$ & 0.5 & -1.095 \\
\hline & $\operatorname{SSG}(n=4)$ & $5.15 \pm 0.11$ & $5.28 \pm 0.12$ & 2.6 & -0.730 \\
\hline & LSG $(n=5)$ & $5.36 \pm 0.13$ & $5.43 \pm 0.09$ & 1.4 & $-2.023 *$ \\
\hline & $\chi^{2}$ & 2.975 & 2.943 & & \\
\hline \multirow{4}{*}{$\mathrm{V}_{40 \mathrm{~m}}\left(\mathrm{~m} \cdot \mathrm{s}^{-1}\right)$} & CG $(n=4)$ & $7.60 \pm 0.34$ & $7.56 \pm 0.28$ & -0.5 & -1.105 \\
\hline & SSG $(n=4)$ & $7.77 \pm 0.16$ & $7.58 \pm 0.18$ & -2.5 & -0.730 \\
\hline & $\operatorname{LSG}(n=5)$ & $7.53 \pm 0.15$ & $7.39 \pm 0.11$ & -1.7 & $-2.032^{*}$ \\
\hline & $\chi^{2}$ & 2.740 & 2.697 & & \\
\hline \multirow{4}{*}{$\mathrm{SL}(\mathrm{m})$} & CG $(n=4)$ & $1.76 \pm 0.07$ & $1.76 \pm 0.10$ & 0.0 & -1.461 \\
\hline & $\operatorname{SSG}(n=4)$ & $1.79 \pm 0.06$ & $1.72 \pm 0.11$ & -4.1 & -0.535 \\
\hline & LSG $(n=5)$ & $1.76 \pm 0.11$ & $1.78 \pm 0.09$ & 1.6 & $-2.060^{*}$ \\
\hline & $\chi^{2}$ & 1.157 & 1.016 & & \\
\hline \multirow{4}{*}{$\mathrm{SF}(\mathrm{Hz})$} & CG $(n=4)$ & $4.57 \pm 0.08$ & $4.60 \pm 0.14$ & 0.7 & -0.730 \\
\hline & SSG $(n=4)$ & $4.63 \pm 0.33$ & $4.79 \pm 0.19$ & 3.4 & -0.736 \\
\hline & LSG $(n=5)$ & $4.60 \pm 0.21$ & $4.56 \pm 0.20$ & -0.9 & -0.542 \\
\hline & $\chi^{2}$ & 0.057 & 2.772 & & \\
\hline \multirow{4}{*}{ TSL (m) } & CG $(n=4)$ & $39.66 \pm 0.25$ & $39.55 \pm 0.18$ & -0.3 & -0.730 \\
\hline & SSG $(n=4)$ & $38.98 \pm 0.95$ & $39.03 \pm 0.36$ & 0.1 & -0.730 \\
\hline & LSG $(n=5)$ & $39.59 \pm 0.47$ & $39.18 \pm 0.62$ & -1.0 & -0.674 \\
\hline & $\chi^{2}$ & 2.390 & 2.196 & & \\
\hline \multirow{4}{*}{ TSN (n) } & CG $(n=4)$ & $23 \pm 1$ & $23 \pm 1$ & 0.0 & -1.414 \\
\hline & $\operatorname{SSG}(n=4)$ & $22 \pm 2$ & $23 \pm 1$ & 4.6 & 0.000 \\
\hline & LSG $(n=5)$ & $23 \pm 2$ & $22 \pm 1$ & -4.3 & -1.732 \\
\hline & $\chi^{2}$ & 1.350 & 2.390 & & \\
\hline
\end{tabular}

Table 2 - Pre-and post-training means, standard deviation, change of rate (\%), and differences in sprint parameters of intergroup and intragroup 
Sport Science Review, vol. XXV, No. 3-4, September 2016

\begin{tabular}{|c|c|c|c|c|c|}
\hline $\begin{array}{l}\text { Vertical jump } \\
\text { parameters }\end{array}$ & Groups & Pre & Post & $\begin{array}{l}\text { Change of } \\
\text { rate }(\%)\end{array}$ & Z \\
\hline \multirow{4}{*}{$\mathrm{SJ}(\mathrm{cm})$} & $C G(n=4)$ & $40 \pm 3$ & $37 \pm 1$ & -5.7 & -1.890 \\
\hline & $\operatorname{SSG}(n=4)$ & $37 \pm 3$ & $35 \pm 3$ & -4.8 & -1.633 \\
\hline & $\operatorname{LSG}(n=5)$ & $39 \pm 6$ & $38 \pm 5$ & -1.5 & -0.378 \\
\hline & $\chi^{2}$ & 0.730 & 1.906 & & \\
\hline \multirow{4}{*}{$\mathrm{CMJ}(\mathrm{cm})$} & $C G(n=4)$ & $43 \pm 2$ & $41 \pm 2$ & -4.7 & -1.841 \\
\hline & $\operatorname{SSG}(n=4)$ & $40 \pm 3$ & $40 \pm 5$ & 0.0 & 0.368 \\
\hline & $\operatorname{LSG}(n=5)$ & $42 \pm 9$ & $42 \pm 9$ & 0.0 & -0.677 \\
\hline & $\chi^{2}$ & 0.941 & 0.217 & & \\
\hline \multirow{4}{*}{$\mathrm{CMJ}-\mathrm{SJ}_{\mathrm{dif}}(\mathrm{cm})$} & $C G(n=4)$ & $3 \pm 1$ & $4 \pm 1$ & 31.3 & 0.000 \\
\hline & $\operatorname{SSG}(n=4)$ & $3 \pm 1$ & $5 \pm 2$ & 58.3 & -1.342 \\
\hline & $\operatorname{LSG}(\mathrm{n}=5)$ & $3 \pm 3$ & $4 \pm 3$ & 31.3 & -1.512 \\
\hline & $\chi^{2}$ & 0.564 & 0.580 & & \\
\hline \multirow{4}{*}{ TDJH (cm) } & $C G(n=4)$ & $44 \pm 6$ & $46 \pm 9$ & 5.7 & -0.557 \\
\hline & $\operatorname{SSG}(n=4)$ & $44 \pm 3$ & $55 \pm 7$ & 25.7 & -1.604 \\
\hline & $\operatorname{LSG}(n=5)$ & $41 \pm 7$ & $40 \pm 6$ & -2.4 & 0.000 \\
\hline & $\chi^{2}$ & 0.639 & $6.031 *$ & & \\
\hline
\end{tabular}

Data are means \pm SD. ${ }^{*} \mathrm{p}<0.05$.

Table 3 - Pre- and post-training means, standard deviation, change of rate (\%), and differences in vertical jump parameters of intergroup and intragroup 
Effects of stride length and frequency training

\begin{tabular}{|c|c|c|c|c|c|}
\hline $\begin{array}{l}\text { Horizontal } \\
\text { jump } \\
\text { parameters }\end{array}$ & Groups & Pre & Post & $\begin{array}{l}\text { Change of } \\
\text { rate }(\%)\end{array}$ & Z \\
\hline \multirow{5}{*}{ SLJ (m) } & CG $(n=4)$ & $2.76 \pm 0.16$ & $2.75 \pm 0.20$ & -0.1 & -0.365 \\
\hline & $\operatorname{SSG}(n=4)$ & $2.80 \pm 0.17$ & $2.80 \pm 0.20$ & 0.0 & -0.365 \\
\hline & & & & & \\
\hline & $\operatorname{LSG}(\mathrm{n}=5)$ & $2.67 \pm 0.16$ & $2.76 \pm 0.19$ & 3.1 & $-2.032 *$ \\
\hline & $\chi^{2}$ & 1.720 & 0.218 & & \\
\hline \multirow{4}{*}{ STJ (m) } & $C G(n=4)$ & $7.98 \pm 0.86$ & $7.92 \pm 0.87$ & -0.7 & -1.461 \\
\hline & $\operatorname{SSG}(n=4)$ & $8.24 \pm 0.64$ & $8.14 \pm 0.57$ & -1.2 & -1.095 \\
\hline & $\operatorname{LSG}(n=5)$ & $7.93 \pm 0.37$ & $8.23 \pm 0.34$ & 3.7 & $-2.023^{*}$ \\
\hline & $\chi^{2}$ & 1.068 & 1.167 & & \\
\hline \multirow{4}{*}{ SQJ (m) } & CG $(n=4)$ & $13.82 \pm 1.03$ & $13.79 \pm 1.16$ & -0.3 & -0.368 \\
\hline & SSG $(n=4)$ & $14.04 \pm 1.18$ & $13.69 \pm 0.97$ & -2.5 & -1.095 \\
\hline & $\operatorname{LSG}(\mathrm{n}=5)$ & $13.47 \pm 0.74$ & $13.80 \pm 0.78$ & 2.4 & $-2.023^{*}$ \\
\hline & $\chi^{2}$ & 2.440 & 0.086 & & \\
\hline \multirow{4}{*}{ STENJ (m) } & $C G(n=4)$ & $28.57 \pm 2.02$ & $28.30 \pm 2.02$ & -0.9 & -1.826 \\
\hline & $\operatorname{SSG}(n=4)$ & $28.99 \pm 1.41$ & $28.06 \pm 1.06$ & -3.2 & -1.095 \\
\hline & $\operatorname{LSG}(\mathrm{n}=5)$ & $27.80 \pm 1.09$ & $28.42 \pm 1.39$ & 2.3 & $-2.023^{*}$ \\
\hline & $\chi^{2}$ & 2.275 & 0.376 & & \\
\hline
\end{tabular}

Data are means $\pm \mathrm{SD} .{ }^{*} \mathrm{p}<0.0$

Table 4 - Pre-and post-training means, standard deviation, change of rate (\%), and differences in horizontal jump parameters of intergroup and intragroup 
Table 2 showed that there were no significant statistical pre- and posttraining intergroup or intragroup differences in sprint parameters except in the LSG for $\mathrm{t}_{40 \mathrm{~m}}, \mathrm{~V}_{40 \mathrm{~m}}$, and SL $(\mathrm{Z}=-2.023,-2.032,-2.060$, respectively; $\mathrm{p}<0.05)$. Table 3 demonstrated that there were no significant statistical pre- and posttraining intergroup or intragroup differences with respect to the vertical jump parameters except for TDJH (for post-training $\chi^{2}=6.031 ; \mathrm{p}<0.05$ ). Table 4 showed that there were no statistical pre- and post-training intergroup or intragroup differences with respect to the horizontal jump parameters except in the LSG for all horizontal jumps $(Z=-2.032,-2.023,-2.023,-2.023$, respectively; $\mathrm{p}<0.05)$.

\section{Discussion}

The objective of this study was to investigate the effects of a two-week training regimen involving $40 \mathrm{~m}$ of additional short and long running stride workouts over $40 \mathrm{~m}$ of acceleration kinematics in a $100-\mathrm{m}$ sprint and jump parameters of male sprinters in the competition phase.

\section{Sprint Parameters during 40-m Acceleration Phase of 100-m Sprint}

This study demonstrated that there are were significant pre- and posttraining intergroup or intragroup differences in SF, TSL, and TSN related to the $40-\mathrm{m}$ acceleration phase of $100 \mathrm{~m}$ of sprint running. Although there were no significant intergroup differences in $\mathrm{t}_{40 \mathrm{~m}}, \mathrm{~V}_{40 \mathrm{~m}}$, and SL in the acceleration phase, there were significant pre- and post-training differences in these three parameters in the LSG $(Z=-2.023,-2.032,-2.060$, respectively; $\mathrm{p}<0.05)$. Donatti (1996) stated that SL depends on the amount of force applied to the ground and SF depends on the central nervous system's capacity to activate neural stimulation for the generation of greater force. In Donatti's (1996), sprinters trained by performing a training regimen focused on short stride length and high stride frequency showed an increase in both their $60-\mathrm{m}$ sprint time (from $6.30 \mathrm{~s}$ to $6.94 \mathrm{~s}$ ) and TSN (from 28.5 strides to 38 strides). In the same study, sprinters trained by performing a training regimen focused on long stride length and low stride frequency showed an increase in their 60-m sprint time (from 6.30s to $6.89 \mathrm{~s}$ ) but a decrease in their TSN (from 28.5 strides to 24.6 strides). In the present study, sprinters were trained by running an additional $40 \mathrm{~m}$ of sprints with short stride length or high stride frequency (SSG or LSG). Donatti's (1996) study is supported by the results of the present study insomuch as $t_{40 \mathrm{~m}}$ and TSN showed an increase ( $5.15 \mathrm{~s}$ vs. $5.74 \mathrm{~s}, 22$ strides vs. 28 strides; respectively) in the SSG. Moreover, $\mathrm{T}_{40 \mathrm{~m}}$ increased from 5.36s to $6.55 \mathrm{~s}$ and TSN decreased from 23 strides to 17 strides in the LSG. Korchemny (1992) used a similar training method for four months and demonstrated that the stride number decreased (7.5-8 to 6.5-7) in the starting acceleration phase (10 m). Korchemny (1992) has 
also showed that 20-m and 55-m sprint times improved with stride frequency $(4.2 \mathrm{~Hz}$ vs. $4.4 \mathrm{~Hz}$ ) but showed no change with stride length. Therefore, it should be noted that this training regimen for sprint running is effective for the acceleration phase. In this study, there were no significant differences in SF and TSN. The results show that SF and TSN increased by $3.4 \%(4.63 \mathrm{~Hz}$ to $4.79 \mathrm{~Hz})$ and $4.6 \%$ (22 strides to 23 strides), respectively in the SSG. At the same time, TSN decreased by $4.3 \%$ (23 strides to 22 strides) in the LSG. Because the study was carried out at the high ability level of Turkish sprinters, sprinters with highstride characteristics could be expected to have participated in the present study. It seems clear that even a change of one stride affects sprint mechanics in the $40-\mathrm{m}$ acceleration phase crucial to the $100-\mathrm{m}$ sprint. On the other hand, a twomicrocycle training regimen involving an additional short stride and long stride may not have an effect on $\mathrm{t}_{40 \mathrm{~m}}$ or $\mathrm{V}_{40 \mathrm{~m}}$ of sprinters in the acceleration phase. When running speed is increased, the contact time decreases. Therefore, the most important difference between high-level sprinters and low-level sprinters is that the former apply more force to the ground and have a shorter contact time. Hence, these sprinters have the ability to reach high speeds with increased SL but the same SF (Weyand et al., 2000). Moreover, another study (Murphy et al., 2003) has demonstrated that rugby and football players exhibit elevated SF but do not experience a change in their SL due to their short CT in the initial acceleration phase. The results of Weyand et al. (2000) are supported by those of Murphy et al. (2003) indicating that sprinters, who can accelerate more rapidly, have short CTs. Football and other sports involve acceleration and changes in direction over short distances by a short sprint running without reaching maximum speed; however, sprinters need a longer acceleration time to reach their maximum speed. Therefore, it is reasonable to observe a change in SF in sports such as football and a change in SL in sprinting. The majority of increased movement in sprinting is afforded by flexion. An increase in hip flexion ensures less time for backward leg movement and quicker recovery (Mann \& Hagy, 1980). Sprinters need much more time to initiate a clawing effect on the track after a powerful push off the ground for rapid leg recovery (Bushnell \& Hunter, 2007). Faster leg recovery based on the direction of leg movement ensures a suitable position from which to accelerate the opposing leg before the foot contacts the ground. Because greater hip flexion provides longer stride opportunities to the legs, sprinters show lower levels of extension in rapid leg strides, which allows the feet to generate a powerful pushing force against the ground. Greater hip flexion also provides longer stride opportunities to the legs because the leg is positioned at the front of the body. Thus, long stride running workouts, which cause greater hip flexion in sprint strides, can help to improve sprint running performance. 


\section{Jump Parameters}

Although no significant differences in SJ and CMJ were observed in this study, there were decreases in their rate of change. Kale et al. (2009) reported that SJ and CMJ showed significant relationships with maximal speed. Bret et al. (2002) observed that a significant relationship between CMJ and acceleration. Sprinters whose explosive power has the most important effect in the acceleration part of their sprint running should reach their body mass from starting speed to maximal speed. SJ performance is characterised by concentric muscle contraction. A sprinter leans forward at the beginning of the acceleration phase to apply force to the ground, which is caused more by elastic responses than concentric muscle contraction (Delecluse, 1997). In the present study, although CMJ decreased for the CG after training, there were no changes in the SSG and LSG. In terms of SJ, all groups showed decreases. These results indicate that sprinters in the SSG and LSG maintained their elastic power. Nagano et al. (2007) reported that maximum strength development through weight training can be achieved by focusing on muscles around the knee joint, performing combined jumping workouts and learning how to use these strengthened muscles to improve athletes' jump performance. In the present study, it was observed that sprinters are stressed by this type of training in the competition phase. Gehri et al. (1998) observed that jumping ability, developed by CMJ or DJ training, provides more contractile rather than elastic muscle function due to higher positive energy production. Because the heel of the foot does not touch the floor immediately after landing on the floor, the depth jump height should be unique to an individual, and an athlete should exhibit optimum jump performance due to the short take-off time (Nagano et al., 2007). Markovich (2007) reported a plyometric improvement of $4.7 \%$ in SJ and $8.7 \%$ in DJ. The results of the present study vary from those reported by Markovich (2007). Competition phase training could be the reason for these differences due to individual training effects. Progressive intensive jump or sprint training can develop musculo-tendon complex elasticity and supportive composition of the body via the ability to energy storage (Elliott et al., 2007). Kubo et al. (2000) observed connective tissue adaptation and an increase in elasticity as a result of this high force development training. Muscles whose function is tailored to certain sports are trained with exercises that improve stretch shortening cycle. Plyometric training is an exercise method involving stretch shortening cycles and jumping movement. Malisoux et al. (2006) observed increases of $12 \%$ in muscle strength and 13\% in vertical jump height after eight weeks of training involving 5228 plyometric jumps; thus, plyometric jumps are effective for stretch shortening exercise performance. Bosco and Komi (1979) documented that plyometric training involves loadings consisting of concentric contractions after eccentric loading and thus gives rise to the stretch reflex, muscle elasticity, and neuromuscular adaptation to the Golgi tendon organ (GTO). The stretch 
reflex starts in the eccentric phase, and therefore, it can help increase motor unit recruitment in the concentric phase. Elastic power is stored in serial and parallel components of muscle connective tissue. When the power is transferred rapidly in the concentric phase, additional force is generated. The GTO serves a protective function against loads, including extra tension in the muscle; however, Hutton et al. (1992) reported that plyometric training caused the loss of sensation of the GTO and gave muscle the potential to overcome greater tension of elastic elements. The present study shows that TDJH increased in the CG and SSG but decreased in the LSG. The difference in results between the SSG and LSG could be explained by the groups' post-training paired comparison of TDJH. $(\mathrm{Z}=-2.262, \mathrm{p}<0.05)$. The rate of change of TDJH also showed a great increase in the SSG. Delecluse et al. (1995) observed that plyometric training involving high velocities without an additional load improved the acceleration phase of sprint running.

There are certain variations to the exercises sprinters can perform in jump training, such as double, single or alternate leg jumps. In the present study, performance in horizontal jump training decreased for all jumps in the CG and SSG but increased in the LSG. Additional long stride training may be the reason for the increase observed for the LSG in this mesocycle.

In the present study, changes in acceleration kinematics parameters over the 40-m acceleration phase of a 100-m sprint and the physiological responses due to additional stride workouts over a two-week training period (a mesocycle in this study) of the competition phase in male sprinters were examined. Important differences in $\mathrm{t}_{40 \mathrm{~m}}, \mathrm{~V}_{40 \mathrm{~m}}$, and SL were observed for additional long stride running workouts. This result was reinforced by differences in both horizontal jump performance and stride length for sprinters in the LSG. Although there were no significant differences in the SSG related to the rate of change, variation trends were observed for $\mathrm{t}_{40 \mathrm{~m}}$ and $\mathrm{V}_{40 \mathrm{~m}}$ as in the LSG but with the inverse effects observed for SL, SF, TSL, and TSN. It is possible that the number of sprinters in each group and the mesocycle training involving a two-week training period were not sufficient to observe a training effect.

\section{Conclusions}

In conclusion, the results of this study show that there were no significant effects of a two-week training regimen involving additional long and short stride running workouts among male Turkish sprinters in the competition phase; however, long stride running workouts may be useful for sprinters who have short stride sprint characteristics. Further investigations must be carried out to evaluate acceleration kinematics, and jump performance among sprinters for possible changes in additional short and long stride running workouts for different training phases and durations that are most closely linked to functional 
sprint performance parameters.

\section{Acknowledgements}

This present paper was supported by Anadolu University Scientific Research Projects by Project 051326. Special thanks are given to Turkish Athletic Federation for the national team camp for the study, Dr. Alper Aşc1 and Dr. Tahir Hazır for sharing their knowledge and experience, Dr. Sinem Hazır Mavili, Dr. Elvin Onarıcı Güngör, Ela Arıcan Gültekin and Aydın Çetin for their assistance in the preparation of measurement settings and National team sprint coaches for giving permission to the sprinters to participate in the study. The author would like to thank Özgür Koçak for the revision of the English grammar.

\section{References}

Abe, T., Fukashiro, S., Harada, T., \& Kawamoto, K. (2001). Relationship between sprint performance and muscle fascicle length in female sprinters. Journal of Physiological Anthropology and Applied Human Science, 20(2), 141-147.

Bosco, C., Luhtanen, P., \& Komi P.V. (1983). A simple method for measurement of mechanical power in jumping. European Journal Applied Physiology, 50, 273 282.

Bosco, C., \& Komi, P.V. (1979). Potentiation of the mechanical behavior of the Human skeletal muscle through prestretching. Acta Physiologica Scandinavica, 106, 467-472.

Bret, C., Rahmani, A., Dufour, A.B., Messonnier, L., \& Lacour, J.R. (2002). Leg strength and stiffness as ability factors in $100 \mathrm{~m}$ sprint running. Journal of Sport Medicine and Physical Fitness, 42(3), 274-281.

Bushnell, T., \& Hunter, I. (2007). Differences in technique between sprinters and distance runners at equal and maximal speeds. Sports Biomechanics, 6(3), pp.261-268.

Delecluse, C. (1997). Influence of strength training on sprint running performance. Sports Medicine, 24(3), 148-156.

Delecluse, C., VanCopenolle, H., Willems, E., VanLeemputte, M., Diels, R., \& Goris, M. (1995). Influence of high-resistance and high velocity training on sprint performance. Medicine and Science Sports \& Exercise, 27(8), 1203-1209. 
Donatti, A. (1996). Development of stride length and stride frequency in sprint performances. Modern Athlete and Coach, 34(1), 3-8.

Elliott, M.C.C.W., Wagner, P.P., \& Chiu, L. (2007). Power athletes and distance training: physiological and biomechanical rationale for change. Sports Medicine, 37(1), 47-57.

Gehri, D.J., Ricard, M.D., Kleiner, D.M., \& Kirkendall, D.T. (1998). A comparison of plyometric training techniques for improving vertical jump ability and energy production. Journal of Strength and Conditioning Research, 12(2), 85-89.

Hawley, J.A. (2000). Training Techniques for Successful Running Performance. In: Hawley JA, editor. Running. Carlton, Victoria: Blackwell Science, pp.44-57.

Hutton, R.S., \& Atwater, S.W. (1992). Acute and chronic adaptations of muscle proprioceptors in response to increased use. Sports Medicine, 14(6), 406-421.

Kale, M., Aşçi, A., Bayrak, C., \& Açikada, C. (2009). Relationships among jumping performances and sprint parameters during maximum speed phase in sprinters. Journal of Strength and Conditioning Research, 23(8), 2272-2279.

Korchemny, R. (1992). A New concept for sprint start and acceleration training. New Studies in Atbletics, 7(4), 65-72.

Kubo, K., Kanehisa, H., Kawaakami, Y., \& Fukunago, T. (2000). Elasticity of tendon structures of the lower limbs in sprinters. Acta Physiologica Scandinavica, 168(2), 327-335.

Malisoux, L., Francaux, M., Nielens, H., \& Theisen, D. (2006). Stretch-shortening cycle exercises: an effective training paradigm to enhance power output of human single muscle fibers. Journal Applied Physiology, 100(3), 771-779.

Mann, R.A., \& Hagy, J. (1980). Biomechanics of walking, running, and sprinting. American Journal of Sports Medicine, 8(5), 345-349.

Mann, R.V., Kotmel, J., Herman, J., Johnson, B., \& Schultz, C. (1984). Kinematic Trends in Elite Sprinters. In: Terauds J, Barthels K, Kreighbaum E, Mann R, Crakes J. editors. Sports Biomechanics. Del Mar: Academic Publishers, 17-33.

Markovich, G. (2007). Does plyometric training improve vertical jump height? A meta-analytical review. British Journal of Sports Medicine, 41(6), 349-355. 
Mero, A. (1988). Force-time characteristics and running velocity of male sprinters during the acceleration phase of sprinting. Research Quarterly for Exercise and Sport, 59(2), 94-98.

Mero, A., \& Peltola, E. (1981). Neural activation in fatiqued and nonfatiqued conditions of short and long sprint running. Biology of Sports, 6(1), 16-22.

Murphy, A.J., Lockie, R.G., \& Coutts, A.J. (2003). Kinematic determinants of early acceleration in field sport athletes. Journal of Sports Science Medicine, 2, 140150 .

Nagano, A., Komura, T., \& Fukashiro, S. (2007). Optimal coordination of maximal effort horizontal and vertical jump motions - a computer simulation study. Biomedical Engineering OnLine, 6(20), 1-9.

Weyand, P.G., Sternlight, D.B., Bellizli, M.J., \& Wright S. (2000). Faster top running speeds are achieved with greater ground forces not more rapid leg movements. Journal of Physiology, 89, 1991-1999.

Mehmet KALE, PhD is an Assistant Professor in the Department of Coaching Education of Faculty of Sport Sciences at Anadolu University in Eskisehir/Turkey. Kale's research interests lie in the areas training and movement theory, training periodization, adaptation to training, sprint mechanics, sprint physiology, sprint endurance, strength and strength training as well as motion analyzes.

\section{Corresponding address:}

Dr. Mehmet Kale

Department of Coaching Education

Faculty of Sport Sciences

Anadolu University

26555 Eskisehir / Turkey

Email:mkale@anadolu.edu.tr

Caner ACIKADA, PhD is a Visiting Professor in the School of Public Health at Indiana University Bloomington. Acikada's research interests lie in the areas of training and competition, physio-mechanical testing and monitoring of high level athletes, periodization problems in high level sports, physio-mechanical factors affecting high level sport, physio-mechanical adaptation to high level training. 
Effects of stride length and frequency training 